\title{
Steady flow of power-law fluids in a 1:3 planar sudden expansion
}

\author{
S. Dhinakaran ${ }^{1,2, *}$, M.S.N. Oliveira ${ }^{2,3}$, F.T. Pinho ${ }^{4}$ and M.A. Alves ${ }^{2}$ \\ ${ }^{1}$ Departament of Mechanical Engineering, Indian Institute of Technology Indore, \\ PACL Campus, Village Harnia Khedi, Mhow Road, Indore, Madhya Pradesh, INDIA 453441. \\ ${ }^{2}$ CEFT, Departamento de Engenharia Quimica, \\ Faculdade de Engenharia da Universidade do Porto, Rua Dr. Roberto Frias, 4200-465 Porto, Portugal. \\ ${ }^{3}$ Department of Mechanical and Aerospace Engineering, \\ University of Strathclyde, Glasgow G1 1XJ, United Kingdom \\ ${ }^{4}$ CEFT, Departamento de Engenharia Mecânica, \\ Faculdade de Engenharia da Universidade do Porto, Rua Dr. Roberto Frias, 4200-465 Porto, Portugal.
}

\begin{abstract}
The laminar flow of inelastic non-Newtonian fluids, obeying the power-law model, through a planar sudden expansion with a 1:3 expansion ratio was investigated numerically using a finite volume method. A broad range of power-law indices in the range $0.2 \leq n \leq 4$ was considered. Shear-thinning, Newtonian and shear-thickening fluids are analyzed, with particular emphasis on the flow patterns and bifurcation phenomenon occurring at high Reynolds number laminar flows. The effect of the generalized Reynolds numbers (based on power-law index, $n$, and the inflow channel height, $h$ ) on the main vortex characteristics and Couette correction are examined in detail in the range varying from $0.01 \leq R e_{g e n} \leq 600$. Values for the critical generalized Reynolds number for the onset of steady flow asymmetry and the appearance of a third main vortex are also included. We found that the shear-thinning behavior increases the critical $R e_{g e n}$, while shear-thickening has the opposite effect. Comparison with available literature and with predictions using a commercial software (Fluent ${ }^{\circledR B}$ 6.3.26) are also presented and discussed. It was found that both results are in good agreement, but that our code is able to achieve converged solution for a broader range of flow conditions, providing new benchmark quality data.
\end{abstract}

Keywords: Power-law model, planar sudden expansion, steady flow, flow bifurcation, shear-thinning and shear-thickening fluids

\section{Introduction}

When a Newtonian fluid flows at low to moderate Reynolds number in a 2D planar channel and encounters a sudden expansion, flow separation occurs resulting in a pair of symmetric recirculating eddies along the downstream walls. The vortices become asymmetric, but steady, when the Reynolds number $(R e)$ is increased above a certain critical value. With a further increase in Re a third vortex is formed downstream of the smallest

\footnotetext{
${ }^{1}$ Corresponding Author: S. Dhinakaran, sdhina@iiti.ac.in, Tel:+91-732-4240746 , Fax: +91-732-4240 700, M.S.N. Oliveira (monica.oliveira@fe.up.pt) ; M. A. Alves (mmavles@fe.up.pt); F. T. Pinho (fpinho@fe.up.pt).
} 
of the two main vortices [1]. Bifurcation phenomenon, consisting of a transition from symmetric to asymmetric flow, occurs above a critical Reynolds number that depends on the expansion ratio of the planar expansion and the rheology of the fluid. The expansion ratio for a planar geometry is defined as the ratio of height of the outlet channel $(H)$ to the height of the inlet channel $(h)$ and henceforth is denoted as $E R$.

Since the early 1970s there has been a number of experimental studies devoted to the subject of flow bifurcation in channels with a sudden planar expansion. Using laser Doppler anemometry (LDA) Durst et al. [1] examined the Newtonian fluid flow in a 1:3 planar symmetric expansion. In their experiments, two symmetric vortices along the walls of the expansion were observed at $R e=56$. At $R e=114$, flow bifurcation was already observed with vortices of unequal size forming at both salient corners. The experimental measurements of Cherdron et al. (1978) [2] also relied on LDA, but were more comprehensive and explored the flow patterns and instabilities in ducts with symmetric expansions, investigating also the effect of the aspect ratio of the tested geometries. The more recent experimental and numerical study of Fearn et al. [3] in a 1:3 planar expansion showed a similar flow bifurcation at a Reynolds number of 40.5. In contrast to the few experimental investigations, there is a large number of numerical works available in the literature and one of its advantages is that it is possible to investigate truly $2 \mathrm{D}$ flows. In his numerical investigation on planar expansion flows with various expansion ratios, Drikakis [4] found that the critical Reynolds number for the symmetry-breaking bifurcation is reduced when the expansion ratio is increased. Battaglia and Papadopoulos [5] studied the influence of three-dimensional effects on the bifurcation characteristics at low Reynolds number flows in rectangular sudden expansions, in the range of $150 \leq R e \leq 600$. All these experimental and numerical studies were concerned with Newtonian fluids.

In many realistic situations the fluids flowing through flow devices are non-Newtonian and show complex rheological behavior. Specifically, they can exhibit shear-thinning or shear-thickening viscosity depending on the type of fluid and thus it is relevant to investigate the non-Newtonian fluid flow in planar expansions starting with simple rheological models in order to independently assess the impact of specific rheological features upon the flow characteristics. If the non-Newtonian solutions are not too concentrated the flows tend to have a high Reynolds number, even leading to turbulent flow. Since the sudden expansion is a well-known geometry for studies of laminar flow instabilities at high Reynolds numbers, in recent years it has naturally started to attract the attention of researchers in the field of non-Newtonian fluid mechanics wishing to investigate the complex interaction between these bifurcations and fluid rheology, namely viscoelasticity. In non-Newtonian fluid mechanics there are other traditional benchmark flows, such as the 4:1 sudden contraction and the flow around a confined cylinder under $50 \%$ blockage ratio, but these have been devised to address the numerical convergence difficulties in creeping flows of viscoelastic fluids. Incidentally, computations of creeping flow of viscoelastic fluids in sudden expansion geometries were found to be harder to converge than the more classical sudden contraction flow [19]. As we show below, the investigations of power law fluids carried out so far in this geometry provide an incomplete picture, which we aim to address in this work.

The non-Newtonian power-law model is the simplest model for a purely viscous fluid that can represent the behavior of shear-shinning, shear-thickening and Newtonian fluids by varying the parameter of the model, n, 
known as the power-law index. Consequently, it comes as no surprise that several numerical studies in the past were performed using the power-law viscosity model to study the flow of shear-thinning and shear-thickening fluids in planar sudden expansions of various $E R$.

Mishra and Jayaraman [6] examined numerically and experimentally the asymmetric steady flow patterns of shear-thinning fluids through planar sudden expansions with a large expansion ratio, $E R=16$. Manica and De Bortoli [7] studied numerically the flow of power-law fluids in a 1:3 planar sudden expansion for $n=0.5$, 1 and 1.5. They presented the vortex characteristics for these values of $n$ and for $30 \leq R e \leq 125$, and observed that the flow bifurcation for shear-thinning fluids occurs at a critical Reynolds number higher than for Newtonian fluids, and that shear-thickening fluids exhibited the lowest critical Reynolds number. Considering again purely viscous fluids represented by the power-law and Casson models, Neofytou [8] analyzed the transition from symmetric to asymmetric flow of power-law fluids with power-law indices in the range $0.3 \leq n \leq 3$ in a 1:2 planar sudden expansion and also studied the effect of Reynolds number on the flow patterns.

Ternik et al. [9] studied the flow through a 1:3 planar symmetric expansion of non-Newtonian fluids with shear-thickening behavior using the quadratic and power-law viscosity models. They compared the results of both models with those of Newtonian fluids and concluded that the occurrence of flow asymmetry is greatly affected by the shear-thickening behavior. Later, Ternik [10], computed the flow of shear-thinning fluids with power-law indices $n=0.6$ and 0.8 in a 1:3 planar sudden expansion. After the first bifurcation, from a symmetric to asymmetric flow, a second flow bifurcation, marking the appearance of a third vortex, was predicted as the generalized Reynolds number was further increased, with shear-thinning delaying the onset of this second bifurcation. More recently, Ternik [11] revisited the generalized Newtonian flow in a two-dimensional 1:3 sudden expansion using the open source OpenFOAM CFD software. The fluid was again represented by the power-law model with power-law index in the range $0.6 \leq n \leq 1.4$ and the simulations were performed for generalized Reynolds numbers in the range $10^{-4} \leq R e_{g e n} \leq 10$ with the emphasis on the analysis of low Reynolds number flows, below the critical conditions for the onset of the pitchfork bifurcation. Small recirculation, typical of creeping flow (called the Moffatt vortices [12]) were observed for all fluids with shear-thinning behavior reducing the size and intensity of the secondary flow.

Numerical simulations of the flow of power-law fluids in a planar 1:3 sudden expansion using commercial or open source codes were attempted by several authors. It was found that the solution convergence is often a major limitation when utilizing these codes especially when the non-Newtonian behavior is enhanced (large or small $\mathrm{n}$ values for power-law model). For instance, Poole and Ridley [13] used Fluent ${ }^{\circledR}$ software to numerically calculate the development-length required to attain fully developed laminar pipe flow of inelastic power-law fluids and were unable to attain a converged solution for $n<0.4$. Ternik [10] reported that the iterative convergence had become increasingly time consuming with a reduction in power-law index, and for $n<0.6$ no converged solutions were obtained using the OpenFOAM software.

From the aforementioned discussion, it is clear that a comprehensive investigation on the flow of power-law fluids in planar sudden expansions is still lacking for power-law indices below $n=0.6$ and above $n=1.4$ and this is clearly seen in Figure 1. This work aims to fill this gap in the literature using an in-house finite-volume code 
[15]. We present a systematic study of the flow in a 1:3 sudden planar expansion for a wide range of power-law indices, $0.2 \leq n \leq 4$, and generalized Reynolds numbers, $0.01 \leq R e_{g e n} \leq 600$, including data for the Couette correction. The critical generalized Reynolds numbers at which symmetry breaking flow bifurcation occurs is reported and the flow structures in the expansion are visualized using streamline plots. We also compare the results obtained with our in-house code with those calculated using the Fluent ${ }^{\circledR} 6.3 .26$ software using exactly the same meshes and flow conditions. The remainder of this paper is organized as follows: in Section 2 we present the mathematical formulation of the problem, and in Section 3 we discuss the numerical method along with the code validation. The results are presented and discussed in Section 4, and Section 5 summarizes the main conclusions.

\section{Mathematical Formulation}

\subsection{Problem Description}

The problem under study is illustrated schematically in Figure 2(a), which also includes the nomenclature used to refer to the various characteristics lengths of the vortices. A $2 \mathrm{D}$, long, planar channel of width h has a sudden expansion to a second channel of width $\mathrm{H}$, thus defining an expansion ratio $\mathrm{ER}=H / h=3$. The center of the co-ordinate axes system lies at the center of the geometry expansion plane. The downstream channel has a length $L_{E}=200 h$ and the upstream channel length is $L_{C}=50 h$. The inlet of the computational domain is located sufficiently far from the expansion plane in order for the inlet fluid flow to become fully developed well before the expansion. Similarly, the outlet of the channel is located far from the region of interest where the separating flow regions develop and exist.

\subsection{Governing equations}

The flow is considered to be laminar, steady and incompressible and the fluid in the channel flows in the positive $x$-direction. This flow is governed by the continuity equation,

$$
\frac{\partial u}{\partial x}+\frac{\partial v}{\partial y}=0
$$

and the momentum equations, Momentum equations:

$$
\begin{aligned}
& \rho\left(\frac{\partial u}{\partial t}+u \frac{\partial u}{\partial x}+v \frac{\partial u}{\partial y}\right)=-\frac{\partial p}{\partial x}+\left(\frac{\partial \tau_{x x}}{\partial x}+\frac{\partial \tau_{x y}}{\partial y}\right) \\
& \rho\left(\frac{\partial v}{\partial t}+u \frac{\partial v}{\partial x}+v \frac{\partial v}{\partial y}\right)=-\frac{\partial p}{\partial y}+\left(\frac{\partial \tau_{x y}}{\partial x}+\frac{\partial \tau_{y y}}{\partial y}\right)
\end{aligned}
$$


where $u$ and $v$ are the velocity components in the $\mathrm{x}$ and y directions, respectively, $p$ is the pressure and $\rho$ is the density of the fluid. The power-law model is used, and the extra-stress tensor is calculated as

$$
\tau_{i j}=2 \eta(\dot{\gamma}) \mathbf{D}_{i j}
$$

where $D_{i j}=\frac{1}{2}\left(\frac{\partial u_{j}}{\partial x_{j}}+\frac{\partial u_{j}}{\partial x_{i}}\right)$ is therate of deformation tensor (with $u_{i}=u$ or $v$ and $x_{i}=x$ or $y$ for $i=1$ and 2, respectively), and the viscosity function is calculated as

$$
\eta(\dot{\gamma})=K \dot{\gamma}^{n-1}
$$

with $K$ being the consistency index and $n$ the power-law index. Here, the shear rate, $\dot{\gamma}$, is related to the second invariant of the rate of deformation tensor $(\mathbf{D})$ as

$$
\dot{\gamma}=\sqrt{2 \mathbf{D}: \mathbf{D}}=\sqrt{2\left(\frac{\partial u}{\partial x}\right)^{2}+2\left(\frac{\partial v}{\partial y}\right)^{2}+\left(\frac{\partial u}{\partial x}+\frac{\partial v}{\partial y}\right)^{2}}
$$

According to the definition used in previous works with power-law fluid flows $[10,11]$ the generalized Reynolds number used throughout this paper is defined based on the upstream channel height and bulk inlet velocity $(\bar{u})$ as

$$
R e_{g e n}=\frac{6 \rho \bar{u}^{(2-n)} h^{n}}{K[(4 n+2) / n]^{n}}
$$

\section{Numerical procedure and validation}

The governing equations (1)-(3) are solved using an in-house finite volume method and employing the SIMPLEC pressure correction algorithm formulated with the collocated variable arrangement [14]. These equations are integrated in space and time over elementary control volumes, resulting in a set of linearized algebraic equations. The CUBISTA high-resolution scheme [14] was used for discretizing the convective terms in the momentum equations, which is formally of third order accuracy. The central difference scheme was used for the discretization of diffusive terms, while a first-order implicit Euler scheme was used for the time derivatives required by the time-marching procedure used to advance the numerical solution until steady state flow is achieved. We note that we are only interested in steady state simulations and therefore the order of convergence of the transient term is irrelevant since it will vanish when steady-state is approached. The set of linearized algebraic equations are solved using either a symmetric or a bi-conjugate gradient method, respectively for pressure and velocities [15] with preconditioning by LU factorization. Iterations are continued until a divergence-free velocity field is obtained. Convergence is assumed when the normalized summation of the residuals decreased below $10^{-8}$ for all equations, which was checked to be sufficiently low by comparing with the solution obtained with the more stringent convergence criterion of $10^{-10}$.

The computational domain is mapped by block-structured meshes and is partitioned into five blocks as shown in Fig. 2b. In the streamwise direction the grids are stretched/compressed in geometric progression in 
each block, whereas in the transverse direction they are uniform. Grids are finer near the step of the expansion (Fig. 2c) while they are coarser near the inlet and outlet. In order to check the grid dependence on the results, four different grids were used, namely meshes A, B, C and D, as detailed in Table 1 which summarizes the number of grid points $\left(N_{x}, N_{y}\right)$, the factors $\left(f_{x}, f_{y}\right)$ and the maximum and minimum size of the smallest grid point used for each mesh $\left(\Delta x_{\max } / h, \Delta y_{\max } / h\right)$ in this study.

\subsection{Boundary conditions}

At the inlet a uniform velocity field is assumed. Since the inlet channel is very long, the flow will be fully developed well upstream of the expansion plane. At the outlet, vanishing gradients are assumed for velocity $(\partial u / \partial x=\partial v / \partial x=0)$ while pressure is linearly extrapolated from the two upstream cells. On the walls no-slip boundary conditions are applied, $u=0, v=0$.

\subsection{Validation}

Analysis of the vortex characteristics obtained at various Reynolds numbers (cf. Table 2) indicates that nearly grid independent results, to within $0.2 \%$ of the refined Mesh D, could be obtained using Mesh C. As such, the subsequent results were obtained with Mesh C, unless stated otherwise. In order to validate the present code, extensive simulations have been performed and the results are compared with those available in the literature and with Fluent ${ }^{\circledR}$ calculations. In the calculations using Fluent ${ }^{\circledR}$, the convective terms were discretized with the QUICK scheme [16] while the pressure-velocity coupling was enforced using standard PISO algorithm [17]. Table 2 presents the vortex characteristics at $n=0.2,0.6,1,1.4$ and 4 obtained using our numerical code, as well as those obtained using Fluent ${ }^{\circledR}$ for three different characteristic values of the generalized Reynolds numbers representing each of the three different flow regimes. For the lower $R e_{g e n}$ considered, the flow is symmetric, while the other two cases correspond to the asymmetric and the asymmetric with third eddy regimes. The results obtained with our code are in good agreement with those predicted using Fluent ${ }^{\circledR}$ with a maximum percentage error of less than $6 \%$ in the coarse mesh, and becoming more accurate as the meshes are refined. Grid dependence tests carried out at the smallest and largest value of $n$ considered here indicates that a maximum uncertainty of $0.523 \%$ occurs at Mesh $\mathrm{C}$ when compared to mesh $\mathrm{D}$ for $n=0.2$ and $R e_{\text {gen }}=$ 420 which means that the current study with mesh $\mathrm{C}$ is sufficiently fine. Similar is the case for $n=4$ with a maximum percentage difference of $0.72 \%$ at $R e_{g e n}=2.5$ while comparing mesh $\mathrm{C}$ with $\mathrm{D}$.

Plots of the dependence of vortex characteristics $\left(X_{a}\right.$ in case of symmetric flow and $X_{d}$ in case of asymmetric flow with a third eddy, cf. Figure 2 a for definitions) with $\left(\Delta x_{\min } / h\right)$ are presented in Fig. 3 for $n=0.6$ and $n=1.4$. It is clear from Fig. 3 that the solution converges to similar values when the mesh size $\left(\Delta x_{\min } / h\right)$ gradually decreases. We note that for low and high $n$ values Fluent ${ }^{\circledR}$ simulations did not fully converge to the prescribed residual tolerance, as also reported by Poole and Ridley [13].

Figures 4, 5 and 6 compare our results for the power-law fluid flow in the 1:3 planar sudden expansion with those available in the literature. Figure 4 reports the recirculation length $\left(X_{a} / h\right)$ and the separation point $\left(Y_{a} / h\right)$ obtained under creeping flow conditions $\left(R e_{g e n}=0.01\right)$ for $0.6 \leq n \leq 1.4$ and compares them with 
those of Ternik [11] revealing a good agreement. The variation of $X_{a} / h$ and $Y_{a} / h$ for generalized Reynolds numbers in the range $0.01 \leq R e_{g e n} \leq 10$ for $0.6 \leq n \leq 1.4$, shown by lines in Fig. 5, again exhibits an excellent agreement with the data of Ternik [11], which are represented as closed symbols. The present code has further been validated by comparing the characteristic dimensions of vortices $\left(X_{a}, X_{b}, X_{c}\right.$ and $X_{d}$, cf. Fig. 2(a)) at $n=$ 0.6, 0.8 and 1 with those of Ternik [10,11] and Oliveira [18]. These comparisons are shown in the bifurcation plots of Fig. 6 and the agreement is again very good.

\section{Results and Discussion}

In the validation section we showed the good quality of our predictions of the recirculation characteristics of the various separated flow regions for the range of conditions available in the literature for power-law fluids. In this section we present a comprehensive set of new results that extend currently available predictions to a wider range of power-law indices and $R e_{g e n}$ as follows:

- Power-law index, $n$ : $0.2,0.4,0.6,0.8,1,1.4,2,3$ and 4

- Generalized Reynolds number, $R e_{g e n}: 0.01 \leq R e_{g e n} \leq 600$.

\subsection{Flow characteristics under creeping flow conditions}

Streamline plots are presented in Fig. 7 for nearly creeping flow conditions $\left(R e_{g e n} \rightarrow 0\right)$. These calculations were performed at $R e_{g e n}=0.01$ and $0.2 \leq n \leq 4$. Under negligible inertia, the flow is always symmetric with equal sized vortices on either side of the centerline. For compactness only half channel is shown for each flow condition. It is clear that the recirculating eddies are small in size and grow with the power-law index, $n$. This is shown more clearly as the variation of the vortex size $X_{a} / h$ as a function of power-law index in Fig. 4(a) for the creeping flow case $\left(R e_{g e n}=0.01\right)$. When the power-law index, $n$, is varied from 0.2 to 1 a quasi-linear increase in $X_{a} / h$ is observed, but with a further increase in $n$ the rise of, $X_{a} / h$ progressively asymptotes to 0.796 for $n>$ 3. A similar feature is observed for the point of separation $\left(Y_{a} / h\right)$ which is plotted in Fig. 4(b). Under creeping flow conditions $Y_{a} / h$ linearly decreases with an increase in $n$ from 0.2 to 1 and thereafter, asymptotically, tends to a value of 0.54 indicating that the recirculation nearly reaches the step of the expansion (cf. Fig. 7). For comparison, the correlations proposed by Ternik [11] are also displayed in the Figs. 4(a) and 3(b) showing that, as per our finding, his correlations are only valid approximately in the range of $0.6 \leq n \leq 1.4$. We present the following correlations for $X_{a} / h$ and $Y_{a} / h$ derived on the basis of our numerical predictions:

$$
\begin{gathered}
\frac{X_{a}}{h}=0.382+0.415 \tanh (1.142 n-0.775) \\
\frac{Y_{a}}{h}=1.03+0.49 \tanh (-1.72 n+1.13)
\end{gathered}
$$


The correlations are accurate to within $1.1 \%$ and $0.9 \%$ of the numerical predictions for $X_{a} / h$ and $Y_{a} / h$, respectively for the entire range of $n$ considered.

\subsection{Vortex characteristics for non-negligible inertia}

Figure 5(a) shows the variation of recirculation length in the streamwise direction, $X_{a} / h$, with generalized Reynolds number in the range $0.01 \leq R e_{\text {gen }} \leq 10$, for $0.2 \leq n \leq 4$. For reference we have also included data from Ternik [11]. At low values of $R e_{g e n}, X_{a} / h$ is constant for each value of $n$ and increases with $n$. As inertia becomes important $Y_{a} / h$ increases, with the value of $R e_{\text {gen }}$ that marks the onset of inertia driven growth of the recirculation progressively decreasing as $\mathrm{n}$ increases. The variation with $n$ and $R e_{g e n}$ of the separation point, $Y_{a} / h$, at the step of the expansion is presented in Fig. 5(b). For shear-thinning fluids, the separation point moves away from the sharp re-entrant corner, and hence $Y_{a} / h$ increases with decreasing $\mathrm{n}$ at a constant value of $R e_{\text {gen }}$ because the separated flow region weakens as is also clear from the visualization in Fig. 7 . On the contrary, for shear-thickening fluids the separation point moves towards the sharp corner and thus $Y_{a} / h$ approaches the limiting value of 0.5 at large values of $n$. Since an increase in inertia leads to longer and stronger recirculations, it comes as no surprise that regardless of the value of $n$, increasing the Reynolds number leads to flow separation right at the corner. Consequently, increasing $R e_{g e n}$ from 0.01 to 10 , results in a decrease in $Y_{a} / h$ for all fluids and in particular for shear-thinning fluids. For shear-thickening fluids the variation is smaller, since the recirculations are wider and $Y_{a} / h$ is already close to 0.5 , but nevertheless a reduction to 0.5 is also seen as $n$ and $R e_{g e n}$ increase.

As already mentioned, the comparisons between our predictions and those of other authors shown in Figs. 6(a)-(c) are found to be in excellent agreement. This also includes the bifurcations observed in these figures, which are explored in detail below.

The variation of recirculation length downstream of the expansion with the generalized Reynolds number for the entire range investigated numerically is presented in Figs. 8(a)-(d) for different values of the power-law index. Initially, at low generalized Reynolds numbers, the recirculating eddies are symmetric and grow with an increase in the Reynolds number (their lengths along the walls are referred to as $X_{a}$ and $X_{b}$ as shown in Fig.2a). When the generalized Reynolds number reaches a critical value $\left(R e_{\text {gen,cr1 } 1}\right)$ one vortex becomes longer than the other (there is no preferred wall for this to happen, but for the sake of understanding we will consider that $X_{a}>X_{b}$ ). With a further increase in $R e_{\text {gen }}$ a third eddy appears on the side of the smaller of the two original main eddies and is located further downstream. The critical $R e_{g e n}$ at which this third main eddy appears is termed as the second critical Reynolds number, $R e_{g e n, c r 2}$. The distance from the step to the point where the flow separates to form the third eddy is termed $X_{c}$ and the distance from the step to the point where the flow reattaches on the wall is termed $X_{d}$ as sketched in Fig.2a.

For a Newtonian fluid $(n=1)$, the first critical Reynolds number is $R e_{g e n, c r 1}=54.5$. Above this value of $R e_{\text {gen }}$, the longer eddy $\left(X_{a}\right)$ continues to grow in size while the smaller eddy decreases in size $\left(X_{b}\right)$ up to $R e_{g e n} \propto 80$. Beyond this value of $R e_{g e n}, X_{a}$ continues to increase and $X_{b}$ starts to grow linearly with $R e_{g e n}$, but less intensively than the growth of $X_{a}$. At $R e_{g e n}=102.2$, the third eddy appears in agreement with the 
results of Oliveira [18], Ternik [10,11]. Similar to $X_{a}$ and $X_{b}$, the variations of $X_{c}$ and $X_{d}$ with $R e_{g e n}$ are initially nonlinear but then become approximately linear above a certain value of $R e_{g e n}$. Comparing the results of $X_{a}, X_{b}, X_{c}$ and $X_{d}$ for $n=1$ and $n=0.8$, we find in Fig. 8(b) that shear-thinning delays all flow transitions. For $n=0.8$ the onset of asymmetry is delayed to a higher generalized Reynolds number of 74.1 and similarly, the appearance of the third eddy $\left(R e_{g e n, c r 2}\right)$ is also delayed to $R e_{g e n}=158.3$. Further decreasing the values of power-law index, corresponding to stronger shear-thinning, results in further delayed flow transitions, shifting the critical values $\left(R e_{g e n, c r 1}\right.$ and $\left.R e_{g e n, c r 2}\right)$ to higher values as observed in Fig. 8(a, b). For example, for $n=$ 0.4 we obtained $R e_{g e n, c r 1}=180.5$ and $R e_{g e n, c r 2}=484$.

The picture for shear-thickening fluids, shown in Figs. 8(c, d), is consistent with the previous results, but also shows some significant differences, especially for very strong shear-thickening behavior $(n \geq 3)$. In fact, by increasing $n$, the flow bifurcation is now anticipated to lower values of $R e_{g e n}$. The critical value, $R e_{g e n, c r 1}=$ 54.5 found for $n=1$, and it is reduced to $R e_{g e n, c r 1}=30$ for $n=1.4$. Moreover, the shape of the variation of $X_{a}$ and $X_{b}$ with $R e_{g e n}$ beyond $R e_{g e n, c r 1}$ looks different from the Newtonian and shear-thinning cases, although for $n=1.4$ we still observe the initial nonlinear variation and decrease of $X_{b}$ immediately above $R e_{g e n, c r 1}$, followed by an increase. Note also that the onset of the third eddy appears very quickly, with $R e_{g e n, c r 2}$ close to $R e_{g e n, c r 1}$. Also, and unlike the Newtonian and shear thinning fluid cases, the size of third recirculation eddy increases significantly with an increase in $R e_{\text {gen }}$. Increasing the power-law index further to $n=2,3$ and 4 induces flow asymmetry at even lower values of $R e_{\text {gen }}$ and it is interesting to note from Fig. $8(\mathrm{~d})$ for $n \geq 3$ that $X_{b}$ becomes nearly constant and no longer increases with $R e_{g e n}$, but instead it starts to reduce in size above $R e_{g e n, c r 2}$. Actually, except for the size of the third eddy, all other characteristics lengths $\left(X_{a}, X_{b}\right.$ and $\left.X_{c}\right)$ decrease with Reynolds number above $R e_{g e n, c r 2}$. Also, the size of the third recirculating eddy is much larger compared to other cases.

The variation of the two critical generalized Reynolds numbers, $R e_{g e n, c r 1}$ and $R e_{g e n, c r 2}$, as a function of $n$ are presented in Fig. 9 where we map different flow pattern types including symmetric, asymmetric and asymmetric with third eddy. In the figure we use different symbols to easily demarcate different flow regimes. It is evident in Fig. 9 that shear-thinning stabilizes the flow by raising significantly the two critical generalized Reynolds numbers, as already pointed out, and also by increasing the difference between the two critical points. In agreement with this, increasing the power-law index above the Newtonian value reduces significantly $R e_{g e n, c r 1}$ and $R e_{g e n, c r 2}$. In fact, for $n=0.4$ we have $R e_{g e n, c r 1}=180.4$ and $R e_{g e n, c r 2}=461.3$, which reduce to $R e_{g e n, c r 1}$ $=5.1$ and $R e_{\text {gen,cr } 2}=5.9$ for $n=3$. We present the following correlations derived based on the calculated numerical data for $R e_{g e n, c r 1}$ and $R e_{g e n, c r 2}$, which are accurate to within $2 \%$ of numerical data:

$$
\begin{gathered}
R e_{g e n, c r 1}=\frac{105}{\sinh (1.5 n)}+\frac{6}{\cosh (0.5 n)} \\
R e_{g e n, c r 2}=\frac{605}{\sinh (3 n)}+\frac{6}{\cosh (n)}
\end{gathered}
$$


To illustrate the flow patterns in different regimes, the streamline plots are depicted in Fig. 10 for shearthinning and in Fig. 11 for Newtonian and shear-thickening fluids at three different generalized Reynolds numbers pertaining to the symmetric, asymmetric and third eddy regimes. Since there is a strong variation of $R e_{g e n, c r 1}$ and $R e_{g e n, c r 2}$ with n, the values of generalized Reynolds numbers used in Figs. 10 and 11 are not the same but they are qualitatively the same in the following sense: for the symmetric regime we considered a value of $R e_{g e n}=0.8 R e_{g e n, c r 1}$, for the asymmetric regime we considered a Reynolds number of $R e_{g e n}=0.5\left(R e_{g e n, c r 1}\right.$ $\left.+R e_{g e n, c r 2}\right)$ and for the third eddy regime we used $R e_{g e n}=1.2 R e_{g e n, c r 2}$. For the shear-thinning fluids, with decreasing value of $n$, the sizes of the vortices are found to be larger, whereas for the shear-thickening fluids, the case is reversed. With an increasing value of $n$, comparing the plots at any of the three different regimes one can find that the vortex size slightly increases with increasing $n$, as seen in Fig. 11(a)-(c). Comparing the streamline patterns for the shear-thinning and shear-thickening cases, we observe that the eddies are stretched along the walls with enhanced shear-thickening behavior, while when shear-thinning becomes more pronounced, the eddies are more curved along the walls.

\subsection{Couette correction}

The Couette correction, $C$, represents the excess pressure drop associated with the flow redevelopment at the expansion, normalized with the average fully developed downstream wall shear stress, $\tau_{\text {wall }}$ :

$$
C=\frac{\Delta P-\left(\Delta P_{f d, u c}+\Delta P_{f d, d c}\right)}{\tau_{\text {wall }}}
$$

where, $\Delta \mathrm{P}$ is the real global pressure drop across the expansion, between two points $\mathrm{A}$ and $\mathrm{B}$ far upstream and downstream of the expansion plane, to ensure that they are well within the regions of fully-developed channel flow, $\Delta P_{f d, u c}$ is the estimated pressure drop between point A and the expansion plane assuming fully-developed flow, and $\Delta P_{f d, d c}$ is the estimated pressure drop between the expansion plane and the point $\mathrm{B}$ also assuming fully-developed flow conditions. Figure 12 depicts the variation of Couette correction as a function of $\operatorname{Re}_{\text {gen }}$ in the range $0.01 \leq R e_{g e n} \leq 10$ for different values of $n$. Comparison of the present data with those of Ternik [11] for $0.6 \leq n \leq 1.4$ are found to be in excellent agreement. The Couette correction plateaus for all values of $n$ for $0.01 \leq R e_{g e n} \leq 0.1$, while for $R e_{g e n}>0.1$ the Couette correction starts to decrease with increasing $R e_{g e n}$ and tends to negative values. Direct inspection of Eq. (12) shows that in this case the real pressure loss through the expansion is less than the estimated for fully developed flow, but a more in depth analysis shows the real meaning of a negative $\mathrm{C}$ to be that the pressure recovery as the fluid goes through the expansion actually exceeds the viscous losses. Additionally, we find that for a constant value of of $R e_{g e n}$, increasing $\mathrm{n}$ leads to a decrease in the value of $\mathrm{C}$. For $R e_{\text {gen }}>10$ we find that $\mathrm{C}$ varies linearly but in inverse proportion to $R e_{g e n}$ an indication that the flow is becoming dominated by inertia with a negative slope due to the decrease of the kinetic energy across the expansion plane $\left(\Delta p_{\text {kin }} \propto-u^{2} ; \tau_{\text {wall }} \propto u ; \Delta p_{\text {ext }} / \tau_{\text {wall }} \propto-R e_{\text {gen }}\right.$, where the excess pressure drop $\left(\Delta p_{\text {ext }}\right)$ is the numerator of equation 12). 


\section{Summary and Conclusion}

We have performed a systematic numerical study on the flow of power-law fluids through a 1:3 planar sudden expansion. Using our finite volume code, we were able to obtain convergence for a much wider range of flow conditions than previously attained in numerical works for power-law fluids. Specifically, we obtained results in the power-law index range $0.2 \leq n \leq 4$. We restricted the analysis to such range of $n$, not because there were convergence difficulties, but because real fluids will hardly behave outside this range.

The following are some of the main conclusions of this study:

1. The flow is steady for the whole range of $R e_{g e n}$ and power-law index, $n$, investigated. The flow is strongly dependent on the power-law index, as significant changes in flow behavior occur with varying $n$ values.

2. In the creeping flow limit $(R e \rightarrow 0)$, Moffatt eddies appear and gradually increase in size with increasing $n$ and asymptotically reach a constant value above $n \approx 3$. The separation point, $Y_{a} / h$ moves towards the sharp corner with an increase in the power-law index, and reaches a constant value at high values of $n$.

3. Flow bifurcation is delayed for shear-thinning fluids $(n<1)$ when compared to the Newtonian fluids $(n=$ 1) while this phenomenon occurs earlier in the case of shear-thickening fluids $(n>1)$. Thus, the critical Reynolds number at which asymmetry is observed increases as $n$ decreases.

4. The recirculating eddies along the walls become more stretched as the shear-thickening behavior is enhanced, while they become more curved when the shear-thinning behavior is enhanced.

Additionally we provide benchmark quality data for this wide range of flow and fluid conditions for such properties as the vortex length characteristics $\left(X_{a}, X_{b}, X_{c}, X_{d}\right.$ and $\left.Y_{a}\right)$ and for the two critical Reynolds numbers marking the onset of the first transition from symmetric to asymmetric flow and marking the second transition from asymmetric flow to asymmetric flow with a third vortex.

\section{Acknowledgement}

Dr. S. Dhinakaran, acknowledges the fund received from the Fundação para a Ciencia e a Tecnologia (FCT) through a Post Doctoral grant SFRH/BPD/70281/2010. The authors are grateful to Fundação para a Ciencia e a Tecnologia (FCT) for funding this work through project grant PTDC/EQU-FTT/71800/2006.

\section{References}

[1] F. Durst, A. Melling, J.H Whitelaw, Low Reynolds number flow over a plane symmetrical sudden expansion, J. Fluid Mech., 64 (1974), 111-128.

[2] W. Cherdron, F. Durst and J.H. Whitelaw, Asymmetric flows and instabilities in symmetric ducts with sudden expansions, J. Fluid Mech., 84 (1978), 13-31. 
[3] R. Fearn, T. Mullin, K. Cliffe, Non-linear flow phenomena in a symmetric sudden expansion, J. Fluid Mech., 211 (1990) 595-608.

[4] D. Drikakis, Bifurcation phenomenon in incompressible sudden expansion flows, Phys. Fluids, 9(1) (1997), $77-87$.

[5] F. Battaglia and G. Papadopoulos, Bifurcation characteristics of flows in rectangular sudden expansion channels, J. Fluids Engg., 128 (2006), 671-679.

[6] S. Mishra and K. Jayaraman, Asymmetric ows in planar symmetric channels with large expansion ratio, Int. J. Numerical Methods in Fluids, 38 (2002), 945-962.

[7] R. Manica and A.L. De Bortoli, Simulation of sudden expansion ows for power-law fluids, J. NonNewtonian Fluid Mech., 121 (2004), 35-40.

[8] P. Neofytou, Transition to asymmetry of generalised Newtonian fluid flows through a symmetric sudden expansion, J. Non-Newtonian Fluid Mech., 133 (2006), 132-140.

[9] P. Ternik, J. Marn and Z. Zunic, Non-Newtonian fluid flow through a planar symmetric expansion: Shearthickening fluids. J. Non-Newtonian Fluid Mech., 13 (2006), 136-148.

[10] P. Ternik, Planar sudden symmetric expansion flows and bifurcation phenomena of purely viscous shearthinning fluids, J. Non-Newtonian Fluid Mech., 157 (2009), 15-25.

[11] P. Ternik, New contributions on laminar flow of inelastic non-Newtonian fluid in the two-dimensional symmetric expansion: Creeping and slowly moving flow conditions, J. Non-Newtonian Fluid Mech., 165 (2010), 1400-1411.

[12] H.K. Moffatt, Viscous and resistive eddies near a shar corner, J. Fluid Mech., 18 (1964), 1-18.

[13] R.J. Poole and B.S. Ridley. Development length requirements for fully-developed laminar pipe flow of inelastic non-Newtonian liquids. ASME Journal of Fluids Engineering, 129 (2007), 1281-1287.

[14] M.A. Alves, P.J. Oliveira and F.T. Pinho, A convergent and universally bounded interpolation scheme for the treatment of advection, Int. J. Numer. Methods Fluids, 41 (2003), 47-75.

[15] J. A. Meijerink and H.A. Van Der Vorst, An iterative solution method for linear systems of which the coefficient matrix is a symmetric M-Matrix, Math. Comput., 31 (1977), 148-160.

[16] B. P. Leonard. A stable and accurate convective modelling procedure based on quadratic upstream interpolation, Comput. Meth. Appl. Mech. Engg., 19 (1979), 59-98.

[17] R. Issa. Solution of the implicitly discretized fluid flow equtions by operator splitting. J. Comput. Phys. 62 (1986), 40-65 
[18] P. J. Oliveira, Asymmetric flows of viscoelastic fluids in symmetric planar expansion geometries, J. NonNewtonian Fluid Mech., 114 (2003), 33-63.

[19] R.J. Poole, F.T. Pinho, M.A. Alves and P.J. Oliveira. The effect of expansion ratio for creeping expansion flows of UCM fluids. J. Non-Newt. Fluid Mech., vol. 163 (2009), 35-44. 
Table 1: Computational domain and mesh characteristics of the 1:3 sudden planar expansion geometry.

\begin{tabular}{|c|c|c|c|c|c|c|}
\hline Mesh & Block & $\mathbf{N}_{\mathbf{x}} \times \mathbf{N}_{\mathbf{y}}$ & $f_{x}$ & $\mathbf{f}_{\mathbf{y}}$ & $\Delta \mathrm{x}_{\min } / \mathrm{h}$ & $\Delta y_{\min } / h$ \\
\hline \multirow{5}{*}{ Mesh A } & I & $72 \times 13$ & 0.9531 & 1.0 & \multirow{5}{*}{0.08} & \multirow{5}{*}{0.08} \\
\hline & II & $35 \times 39$ & 1.01960 & 1.0 & & \\
\hline & III & $38 \times 39$ & 1.0000 & 1.0 & & \\
\hline & IV & $63 \times 39$ & 1.0204 & 1.0 & & \\
\hline & V & $75 \times 39$ & 1.0328 & 1.0 & & \\
\hline \multirow{5}{*}{ Mesh B } & I & $144 \times 25$ & 0.9763 & 1.0 & \multirow{5}{*}{0.04} & \multirow{5}{*}{0.04} \\
\hline & II & $70 \times 75$ & 1.0098 & 1.0 & & \\
\hline & III & $76 \times 75$ & 1.0000 & 1.0 & & \\
\hline & IV & $126 \times 75$ & 1.0101 & 1.0 & & \\
\hline & V & $150 \times 75$ & 1.0158 & 1.0 & & \\
\hline \multirow{5}{*}{ Mesh C } & I & $144 \times 25$ & 0.9881 & 1.0 & \multirow{5}{*}{0.02} & \multirow{5}{*}{0.02} \\
\hline & II & $70 \times 75$ & 1.0049 & 1.0 & & \\
\hline & III & $76 \times 75$ & 1.0000 & 1.0 & & \\
\hline & IV & $126 \times 75$ & 1.0051 & 1.0 & & \\
\hline & $\mathrm{V}$ & $150 \times 75$ & 1.0079 & 1.0 & & \\
\hline \multirow{5}{*}{ Mesh D } & I & $576 \times 102$ & 0.9940 & 1.0 & \multirow{5}{*}{0.01} & \multirow{5}{*}{0.01} \\
\hline & II & $280 \times 306$ & 1.0024 & 1.0 & & \\
\hline & III & $304 \times 306$ & 1.0000 & 1.0 & & \\
\hline & IV & $504 \times 306$ & 1.0025 & 1.0 & & \\
\hline & $\mathrm{V}$ & $600 \times 306$ & 1.0039 & 1.0 & & \\
\hline
\end{tabular}


Table 2: Mesh dependence tests and comparisons with Fluent for the same parameters at different $n$.

(a) $\mathbf{n}=\mathbf{0 . 2}$

\begin{tabular}{|l|c|c|c|c|c|c|}
\hline \multicolumn{2}{|c|}{ Present calculations } \\
\hline & \multicolumn{2}{|c|}{$R e_{\text {gen }}=200$} & \multicolumn{2}{|c|}{$R e_{\text {gen }}=420$} & \multicolumn{2}{c|}{$R e_{\text {gen }}=600$} \\
& $X_{a} / h$ & $X_{b} / h$ & $X_{a} / h$ & $X_{b} / h$ & $X_{a} / h$ & $X_{b} / h$ \\
\hline Mesh A & 4.1407 & 4.1407 & 12.6618 & 4.9030 & 19.4215 & 5.7361 \\
Mesh B & 4.1956 & 4.1956 & 12.8926 & 4.9829 & 19.6939 & 5.8058 \\
Mesh C & 4.2451 & 4.2451 & 13.8157 & 5.0231 & 19.7560 & 5.8302 \\
Mesh D & 4.2543 & 4.2543 & 13.8880 & 5.0443 & 19.7775 & 5.8418 \\
\hline
\end{tabular}

(b) $\mathbf{n}=\mathbf{0 . 6}$

\begin{tabular}{|c|c|c|c|c|c|c|c|c|}
\hline \multicolumn{9}{|c|}{ Present calculations } \\
\hline & \multicolumn{2}{|c|}{$R e_{\text {gen }}=90$} & \multicolumn{2}{|c|}{$R e_{g e n}=180$} & \multicolumn{4}{|c|}{$R e_{\text {gen }}=360$} \\
\hline & $X_{a} / h$ & $X_{b} / h$ & $X_{a} / h$ & $X_{b} / h$ & $X_{a} / h$ & $X_{b} / h$ & $X_{a} / h$ & $X_{b} / h$ \\
\hline Mesh A & 4.7456 & 4.7456 & 12.3070 & 4.1773 & 19.6293 & 5.0612 & 17.2218 & 26.6961 \\
\hline Mesh B & 4.8000 & 4.8000 & 12.4731 & 4.2488 & 20.3754 & 5.2192 & 18.0315 & 27.0220 \\
\hline Mesh C & 4.8599 & 4.8599 & 12.5133 & 4.2651 & 20.5289 & 5.2589 & 18.1721 & 27.1130 \\
\hline Mesh D & 4.8652 & 4.8652 & 12.5426 & 4.2815 & 20.5426 & 5.2715 & 18.1926 & 27.1250 \\
\hline \multicolumn{9}{|c|}{ Fluent calculations } \\
\hline Mesh A & 4.6094 & 4.6094 & 12.1364 & 4.0821 & 19.5867 & 4.9382 & 17.6233 & 26.2325 \\
\hline Mesh B & 4.6993 & 4.6993 & 12.3259 & 4.2003 & 20.2350 & 5.1555 & 18.0885 & 26.6610 \\
\hline Mesh C & 4.7843 & 4.7843 & 12.3935 & 4.2388 & 20.4223 & 5.2219 & 18.1769 & 26.8011 \\
\hline Mesh D & 4.8448 & 4.8448 & 12.4256 & 4.2544 & 20.4649 & 5.2425 & 18.1753 & 26.8552 \\
\hline
\end{tabular}

(c) $\mathbf{n}=\mathbf{1}$

\begin{tabular}{|c|c|c|c|c|c|c|c|c|}
\hline \multicolumn{9}{|c|}{ Present calculations } \\
\hline & \multicolumn{2}{|c|}{$R e_{\text {gen }}=50$} & \multicolumn{2}{|c|}{$R e_{\text {gen }}=70$} & \multicolumn{4}{|c|}{$R e_{g e n}=120$} \\
\hline & $X_{a} / h$ & $X_{b} / h$ & $X_{a} / h$ & $X_{b} / h$ & $X_{a} / h$ & $X_{b} / h$ & $X_{a} / h$ & $X_{b} / h$ \\
\hline Mesh A & 5.0633 & 5.0633 & 9.0012 & 3.6573 & 12.7426 & 3.9197 & 11.1772 & 16.7932 \\
\hline Mesh B & 5.0739 & 5.0739 & 9.0291 & 3.6741 & 12.8490 & 3.9551 & 11.2838 & 16.8711 \\
\hline Mesh C & 5.0824 & 5.0824 & 9.0427 & 3.6744 & 12.8674 & 3.9620 & 11.2967 & 16.8950 \\
\hline Mesh D & 5.0873 & 5.0873 & 9.0495 & 3.6742 & 12.8721 & 3.9643 & 11.2647 & 16.9065 \\
\hline \multicolumn{9}{|c|}{ Fluent calculations } \\
\hline Mesh A & 4.8497 & 4.8497 & 8.8466 & 3.5328 & 12.6328 & 3.7859 & 11.6557 & 17.5246 \\
\hline Mesh B & 4.9681 & 4.9681 & 8.9479 & 3.6139 & 12.7920 & 3.8893 & 11.5264 & 16.6664 \\
\hline Mesh C & 5.0361 & 5.0366 & 9.0030 & 3.6461 & 12.8426 & 3.9322 & 11.4155 & 16.7913 \\
\hline Mesh D & 5.0585 & 5.0585 & 9.0011 & 3.6672 & 12.8376 & 3.9452 & 11.3332 & 16.8611 \\
\hline
\end{tabular}


(d) $\mathbf{n}=\mathbf{1 . 4}$

\begin{tabular}{|c|c|c|c|c|c|c|c|c|}
\hline \multicolumn{9}{|c|}{ Present calculations } \\
\hline & \multicolumn{2}{|c|}{$R e_{\text {gen }}=20$} & \multicolumn{2}{|c|}{$R e_{g e n}=40$} & \multicolumn{4}{|c|}{$R e_{g e n}=65$} \\
\hline & $X_{a} / h$ & $X_{b} / h$ & $X_{a} / h$ & $X_{b} / h$ & $X_{a} / h$ & $X_{b} / h$ & $X_{a} / h$ & $X_{b} / h$ \\
\hline Mesh A & 3.6438 & 3.6438 & 8.9890 & 3.5175 & 11.1289 & 3.5898 & 9.1607 & 17.1619 \\
\hline Mesh B & 3.6473 & 3.6473 & 8.9963 & 3.5163 & 11.2032 & 3.5984 & 9.2285 & 17.1161 \\
\hline Mesh C & 3.6519 & 3.6519 & 8.8895 & 3.5190 & 11.2023 & 3.6009 & 9.2261 & 17.1081 \\
\hline Mesh D & 3.6550 & 3.6550 & 9.0006 & 3.5190 & 11.2047 & 3.6246 & 9.2315 & 17.1019 \\
\hline \multicolumn{9}{|c|}{ Fluent calculations } \\
\hline Mesh A & 3.4387 & 3.4387 & 8.7974 & 3.3281 & 10.9914 & 3.4189 & 9.5822 & 16.7157 \\
\hline Mesh B & 3.5376 & 3.5374 & 8.8890 & 3.4352 & 11.1210 & 3.5213 & 9.4434 & 16.8762 \\
\hline Mesh C & 3.5970 & 3.5972 & 8.9042 & 3.4805 & 11.1744 & 3.5674 & 9.3350 & 17.0105 \\
\hline Mesh D & 3.6260 & 3.6260 & 8.9742 & 3.5002 & 10.2272 & 3.6241 & 8.5892 & 17.0685 \\
\hline
\end{tabular}

(e) $\mathbf{n}=\mathbf{4}$

\begin{tabular}{|l|c|c|c|c|}
\multicolumn{2}{|c|}{ Present calculations } \\
& \multicolumn{2}{|c|}{ Re $_{\text {gen }}=0.5$} & \multicolumn{2}{c|}{$R e_{\text {gen }}=2.5$} \\
& $X_{a} / h$ & $X_{b} / h$ & $X_{a} / h$ & $X_{b} / h$ \\
Mesh A & 3.8113 & 3.8113 & 11.1927 & 5.7675 \\
Mesh B & 3.8881 & 3.8881 & 11.5124 & 5.9253 \\
Mesh C & 3.9236 & 3.9236 & 11.6652 & 5.9972 \\
Mesh D & 3.9450 & 3.9450 & 11.7498 & 6.0364 \\
\hline
\end{tabular}


8. Figures

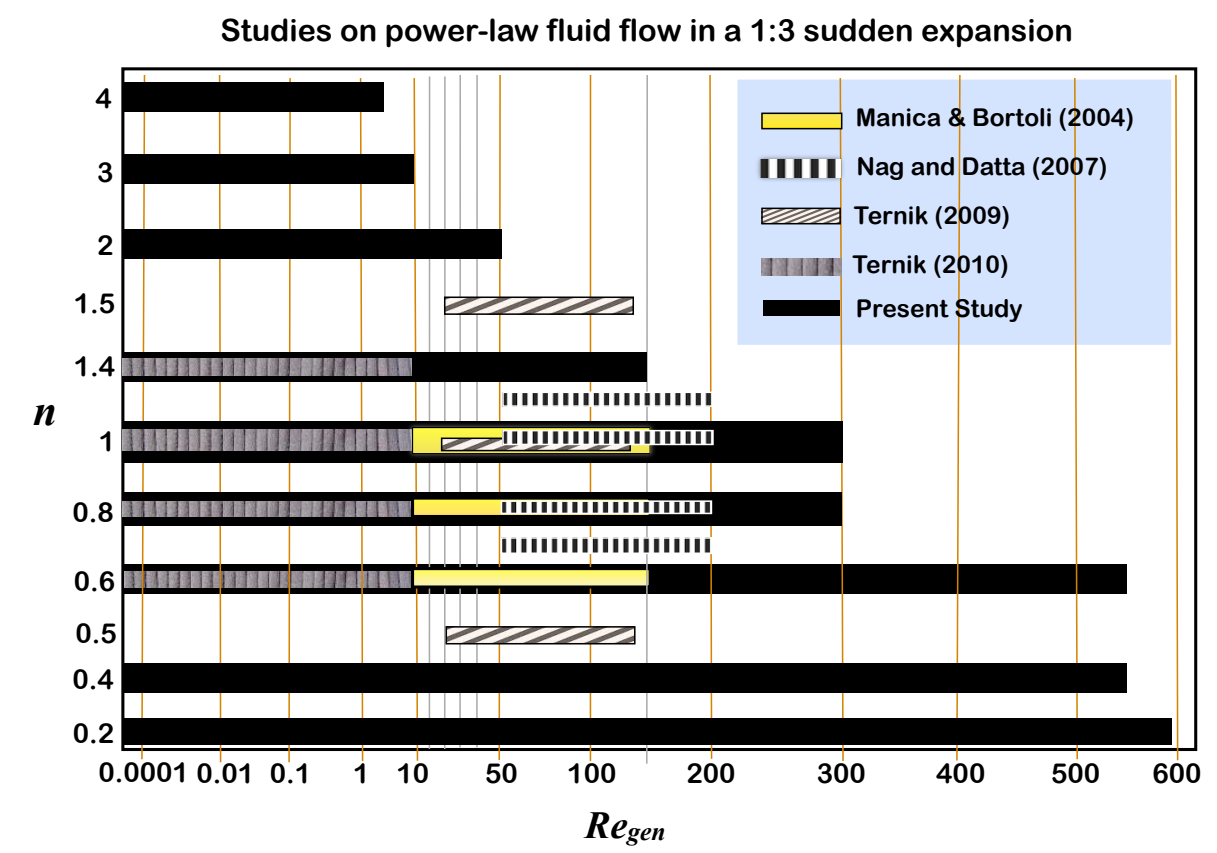

Figure 1: Graphical illustration of the range of generalised Reynolds numbers (Regen and power-law induced $(n)$ used in various studies in the archival literature. 

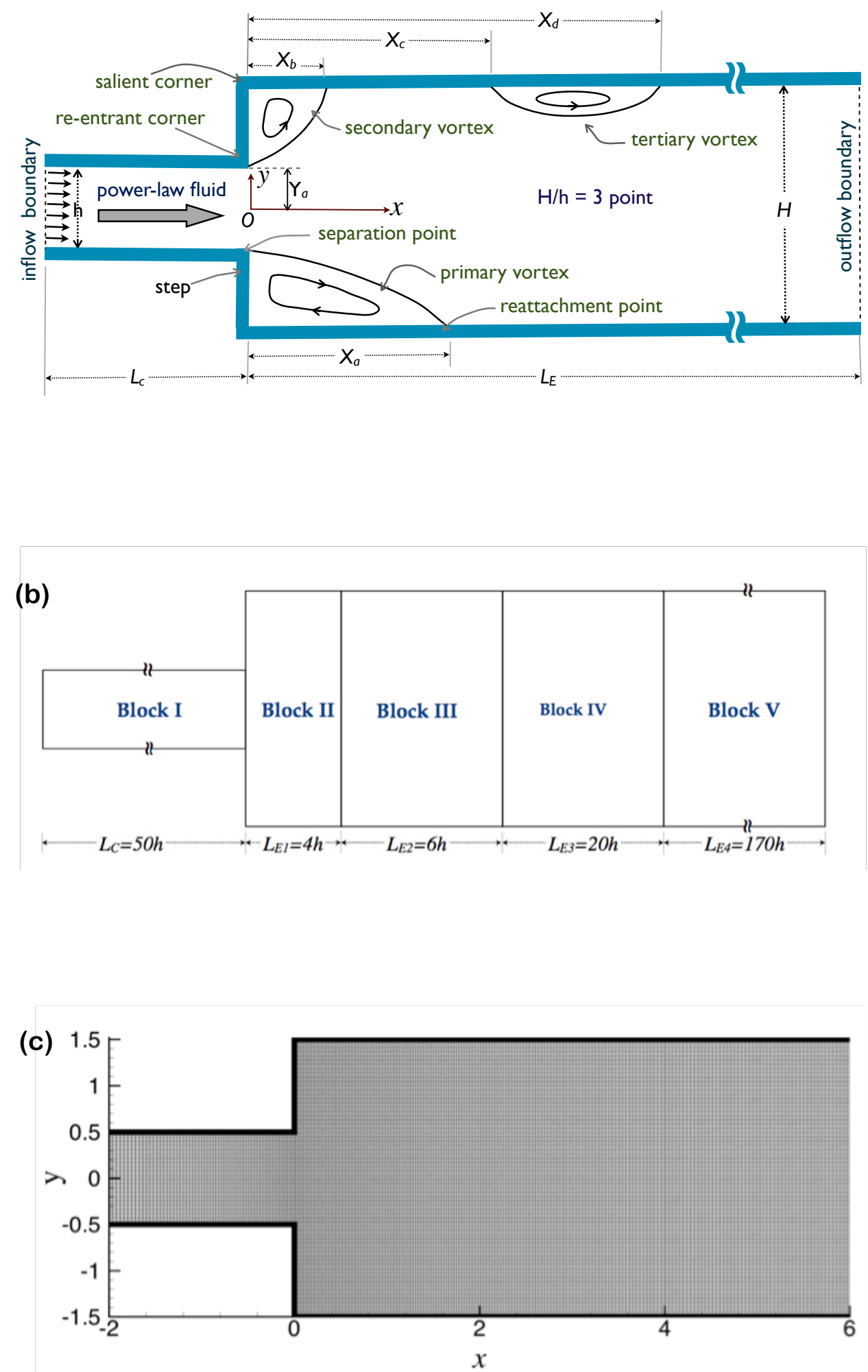

Figure 2: Illustration of (a) two-dimensional 1:3 sudden planar expansion geometry considered in the study and (b) Blocks that were used; (c) Mesh distribution near the expansion plane (Mesh C, $-2 \leq x \leq 6$ and $-1.5 \leq y \leq 1.5$ ). 

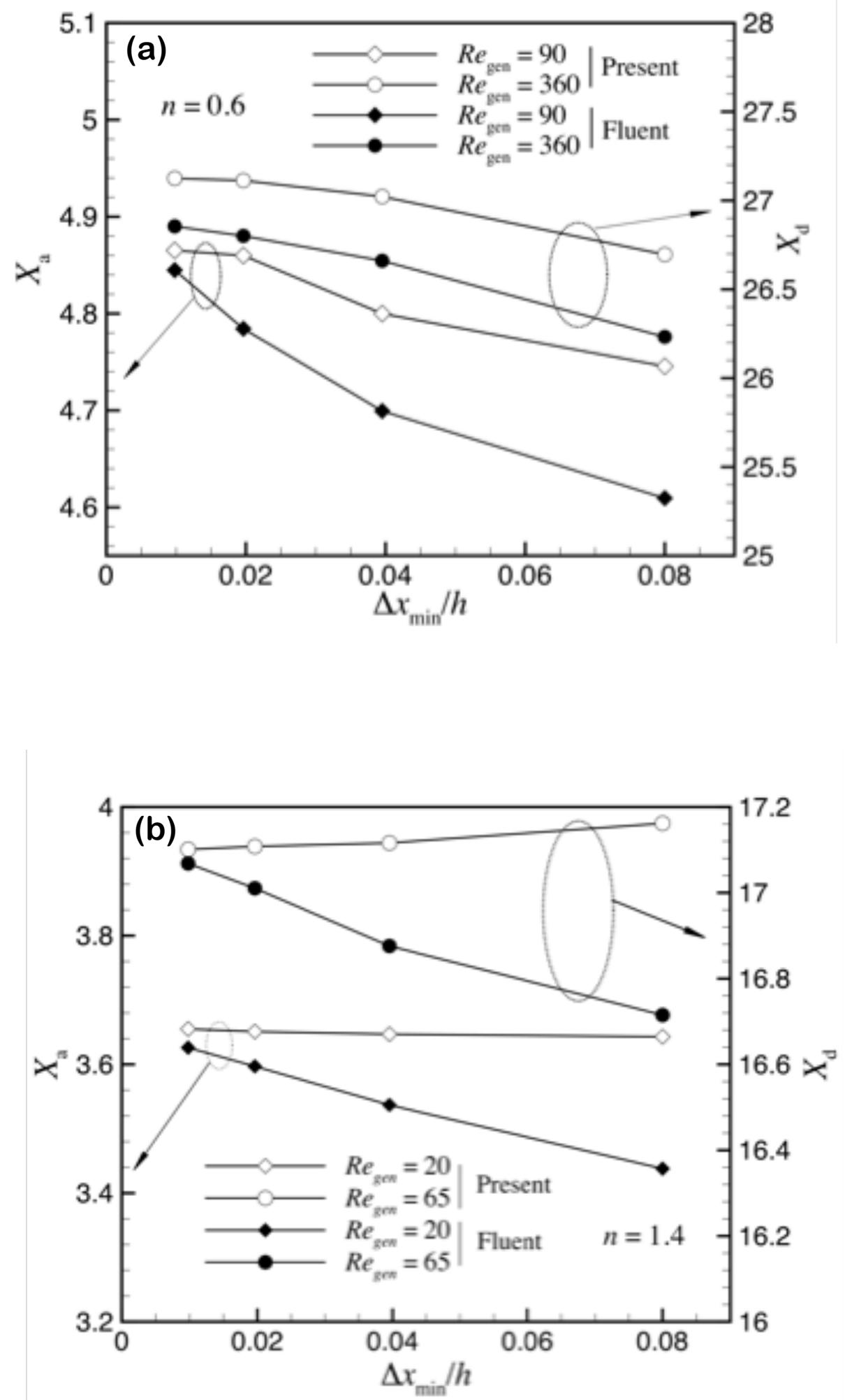

Figure 3: Dependence of vortex characteristics $\left(X_{a}\right.$ and $X_{d}$ in case of symmetric flow and asymmetric flow with a third eddy, respectively) on the smallest grid size $\left(\Delta x_{\min } / h\right)$ corresponding to different meshes obtained with our code and Fluent :(a) $n=$ 0.6 and $(\mathrm{b}) n=1.4$ 

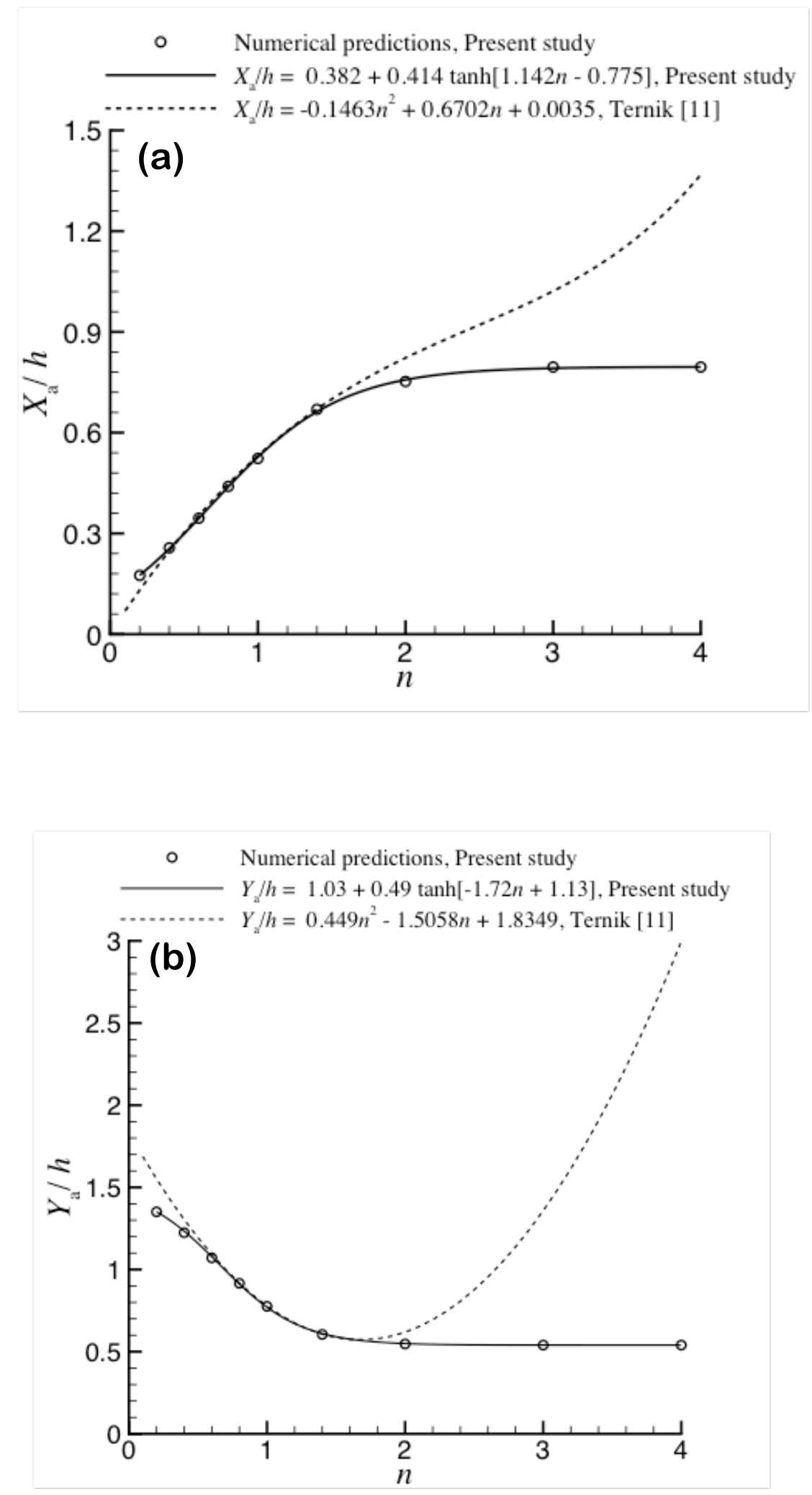

Figure 4: Comparison of present predictions of vortex size with those of Ternik [12] for power-law index in the range $0.6 \leq n \leq 1.4$ at $\operatorname{Re}_{\text {gen }}=0.01$. 

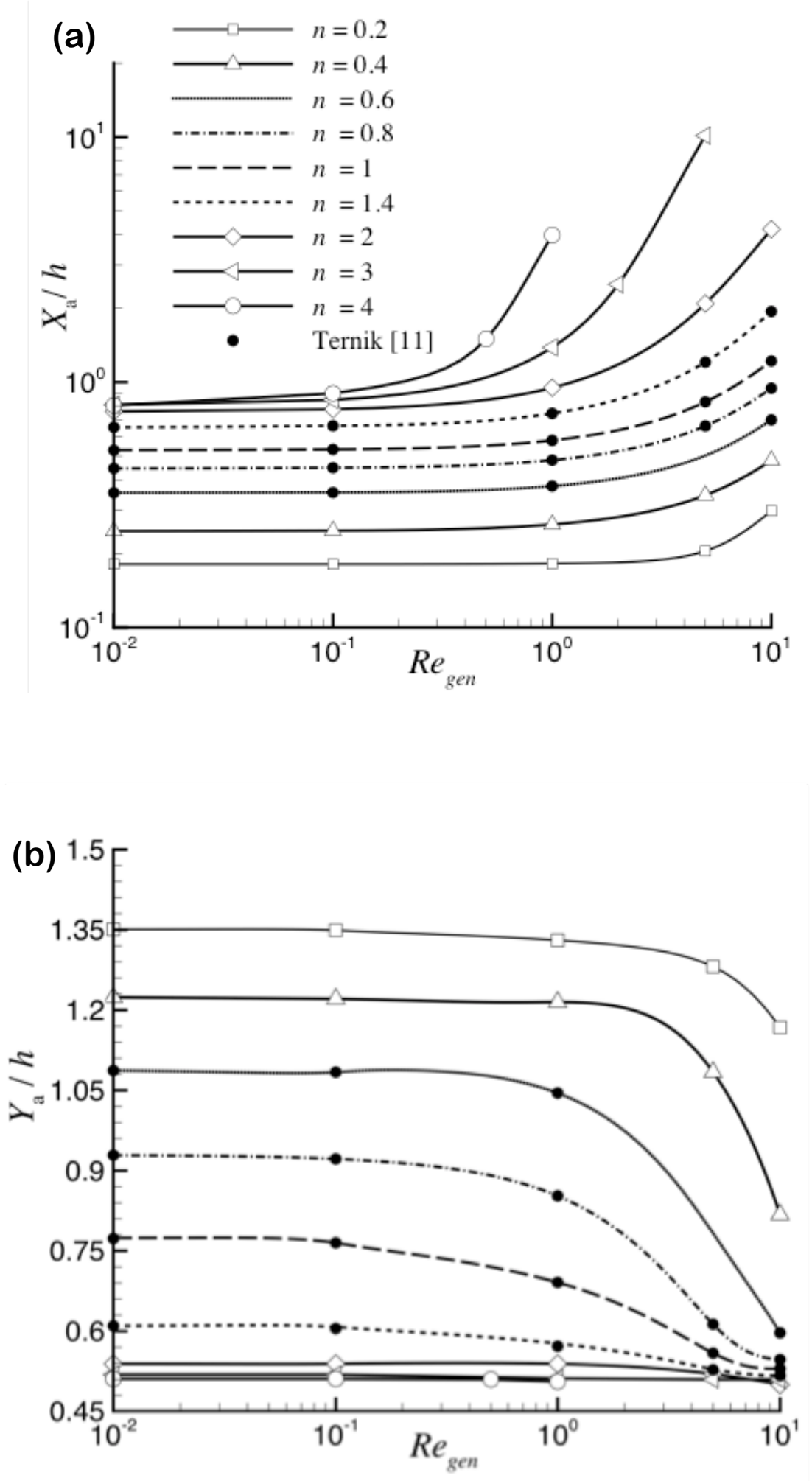

Figure 5: Variation of the main vortex characteristics with the generalized Reynolds number $\left(0.01 \leq R e_{\text {gen }} \leq 10\right)$ at different power-law index in the range $0.2 \leq n \leq 4$ : (a) $X_{a} / h$ and (b) $Y_{a} / h$. Legend in (a) is also applicable to (b). 

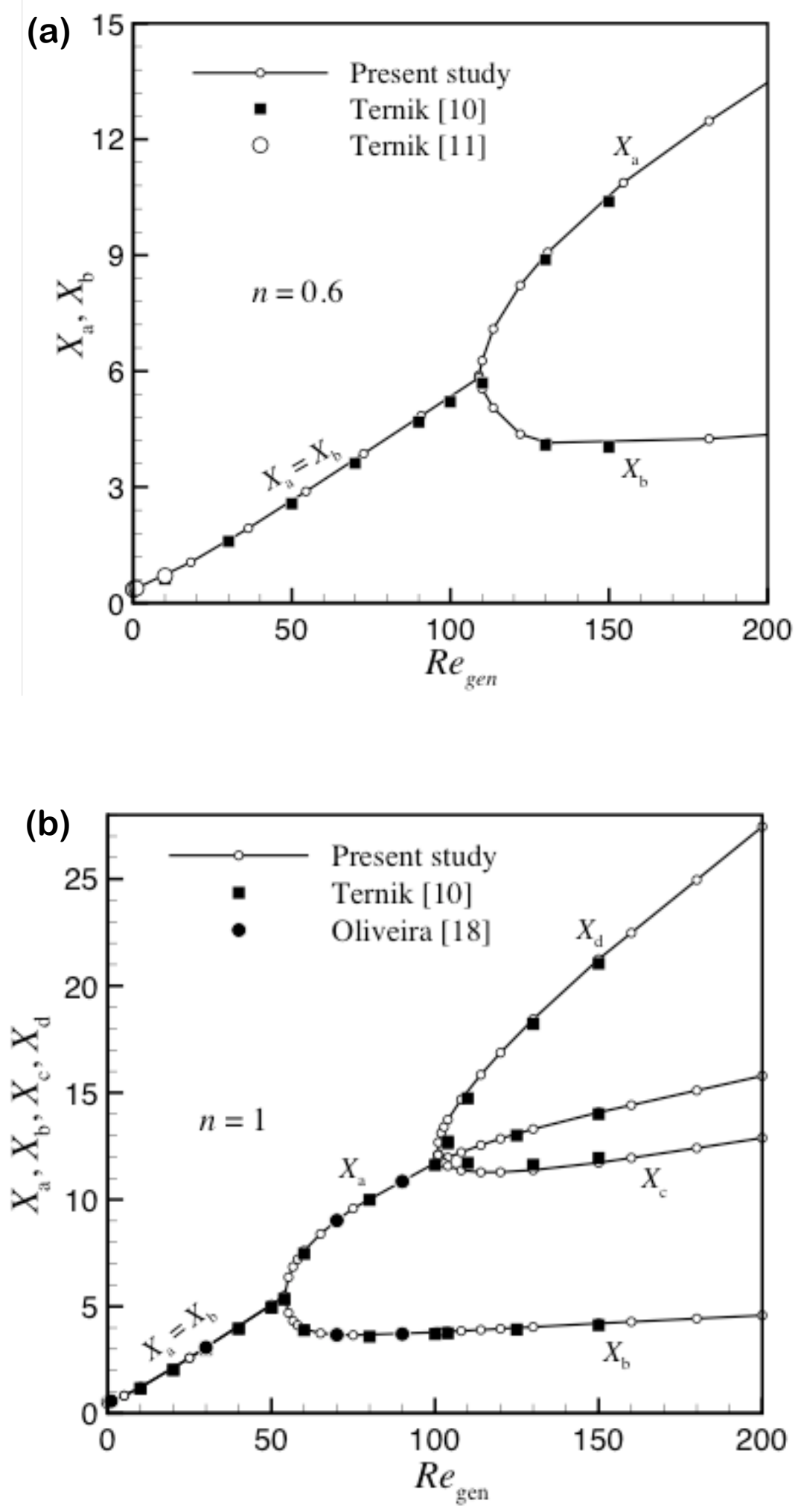

Figure 6: Variation of vortex size with generalized Reynolds number at $n=0.6$ and 1 for the power-law fluid flow in a 1:3 planar sudden expansion and comparison with the available literature data. 

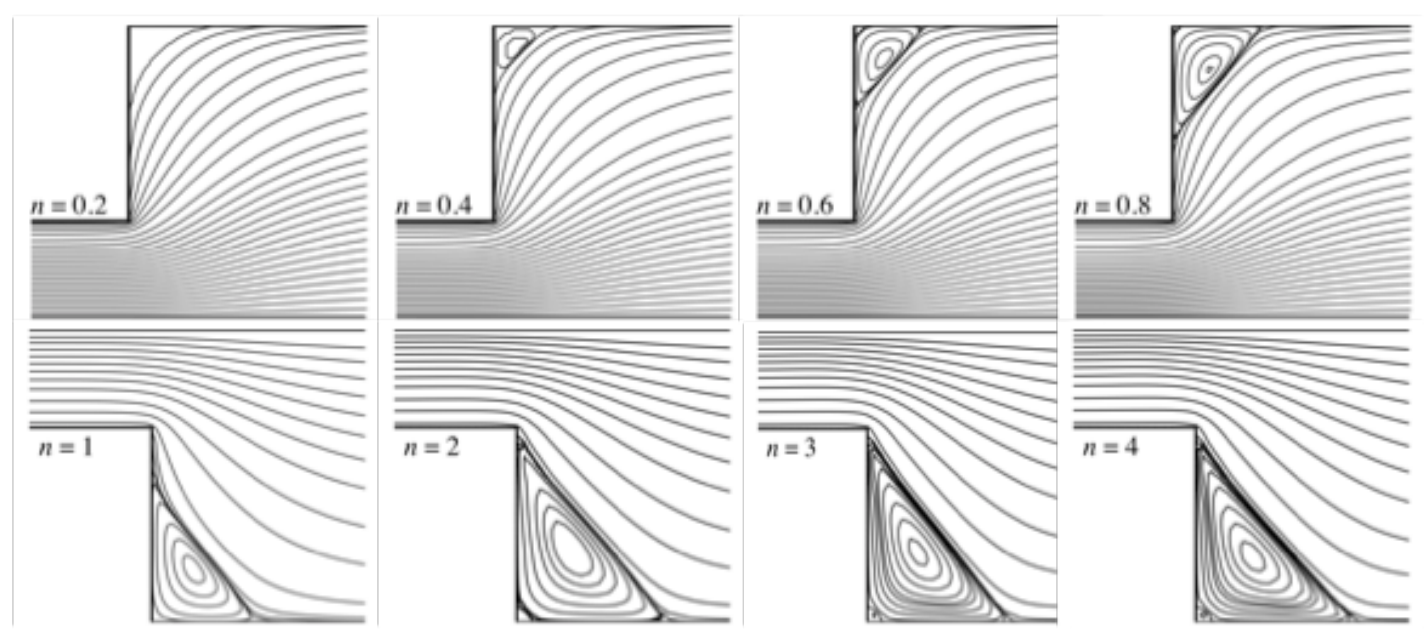

Figure 7: Flow patterns obtained under creeping flow conditions $\left(R e_{g e n}=0.01\right)$ at different power-law index in the range $0.2 \leq n \leq 4$. 

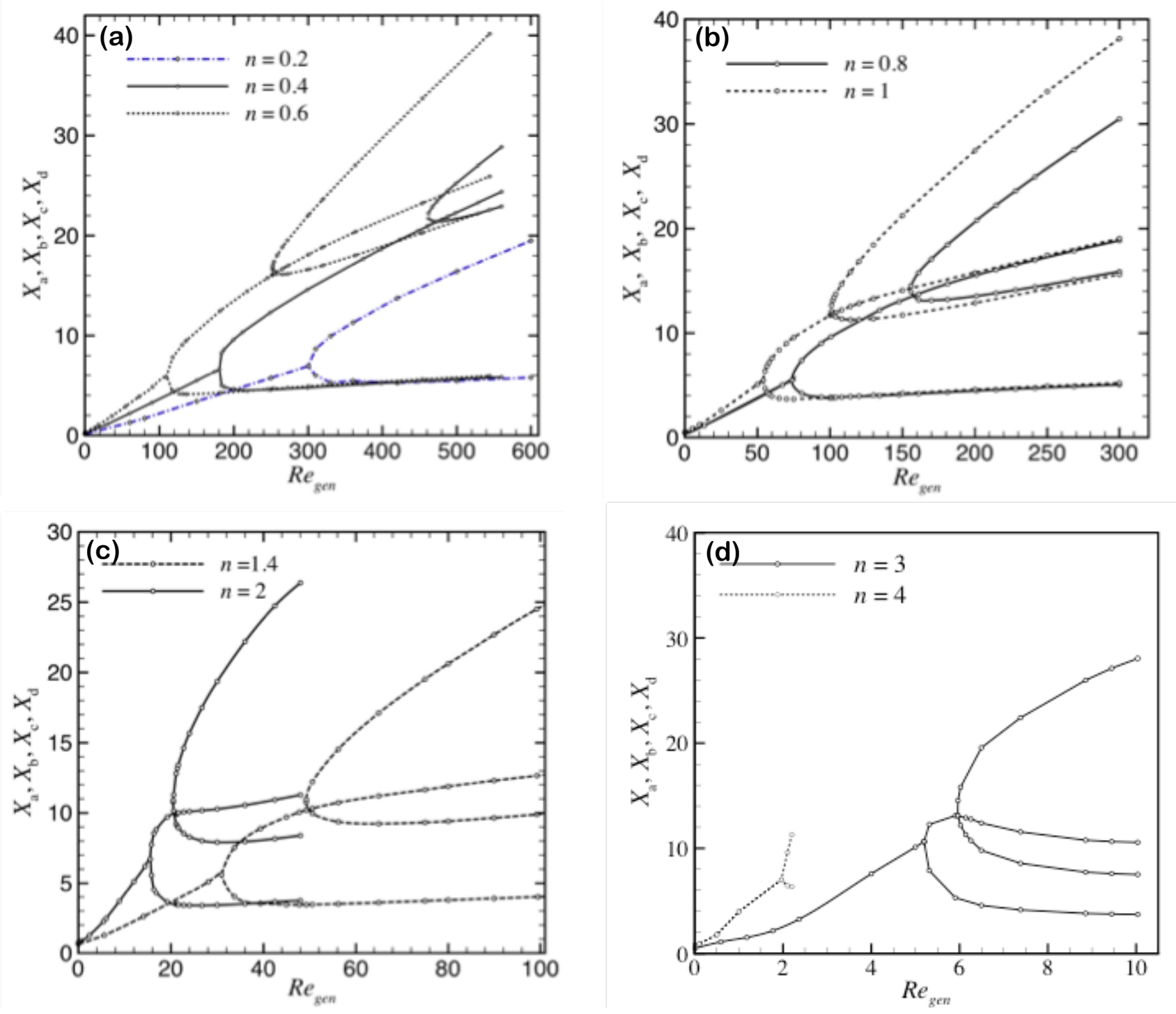

Figure 8: Variation of vortex size with $R e_{\text {gen }}$ at different values of power-law index: (a) $n=0.2,0.4$ and 0.6 ; (b) $n=0.8$ and 1 ; (c) $n=1.4$ and 2 and (d) $n=3$ and 4 . 


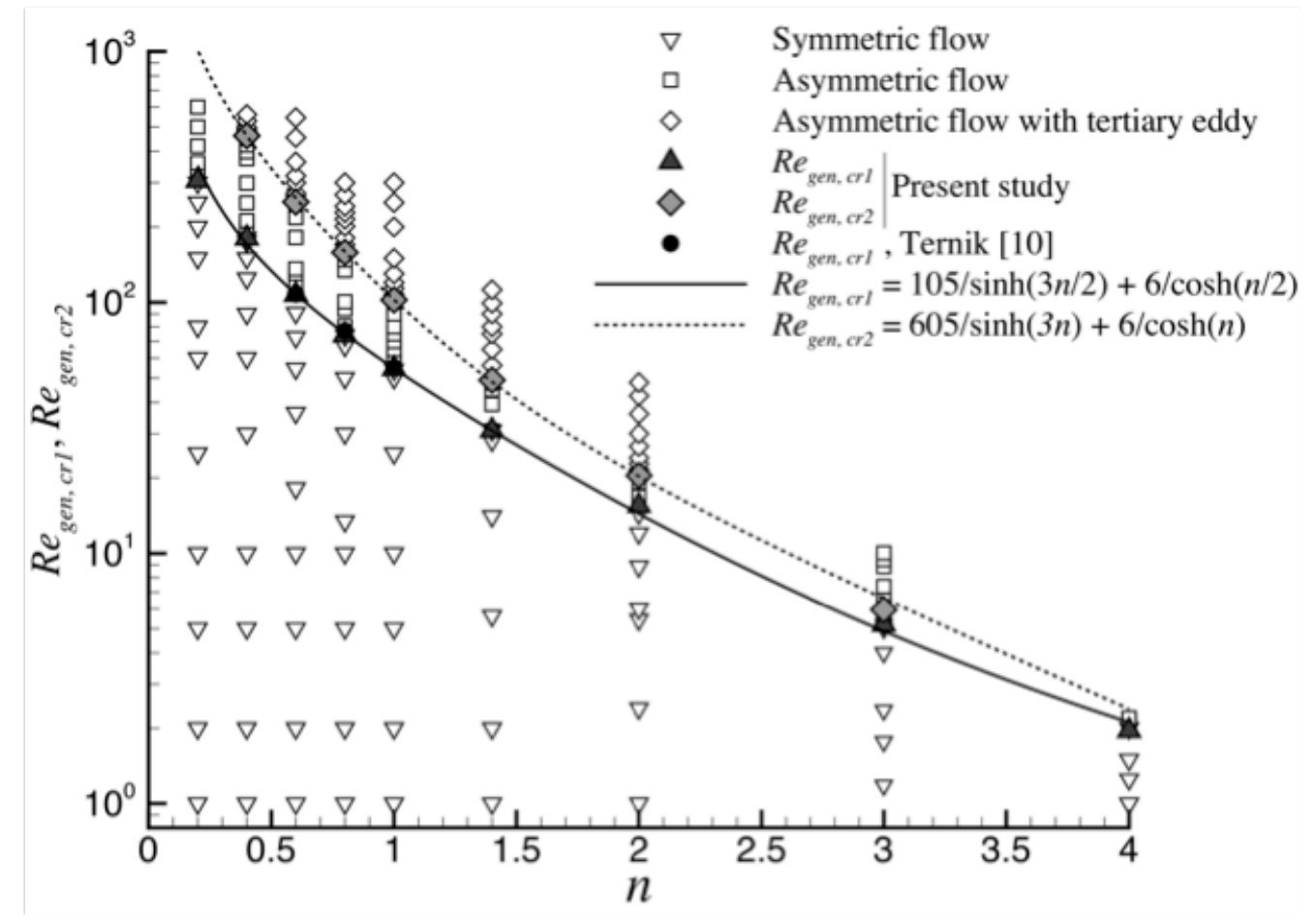

Figure 9: : Effect of power-law index on the critical generalized Reynolds numbers at which flow bifurcation occurs $\left(R e_{g e n, c r 1}\right)$ and at which a tertiary recirculating eddy appears $\left(R e_{g e n, c r 2}\right)$. Maps of the three flow patterns are shown by different symbols. 
(a)

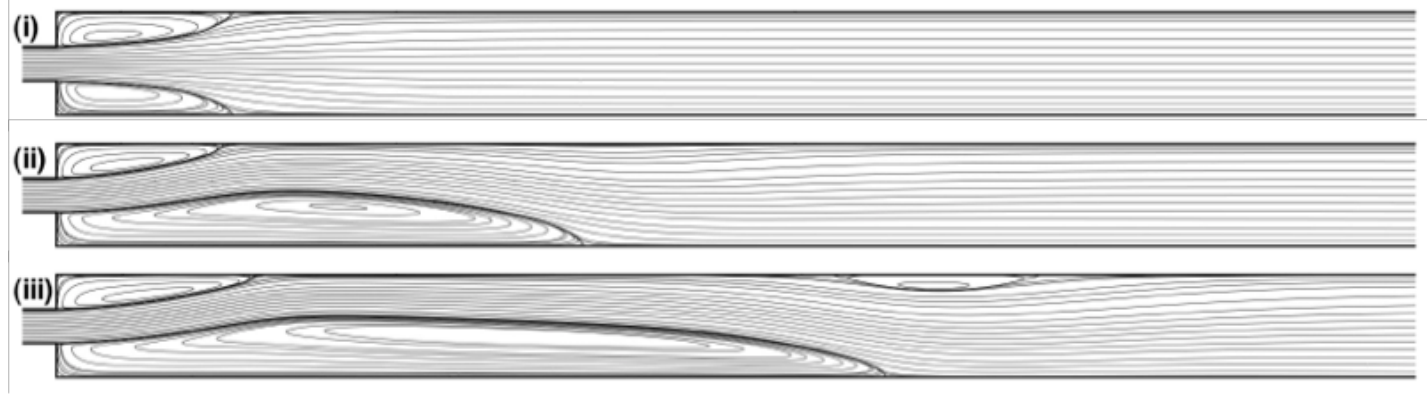

(b)

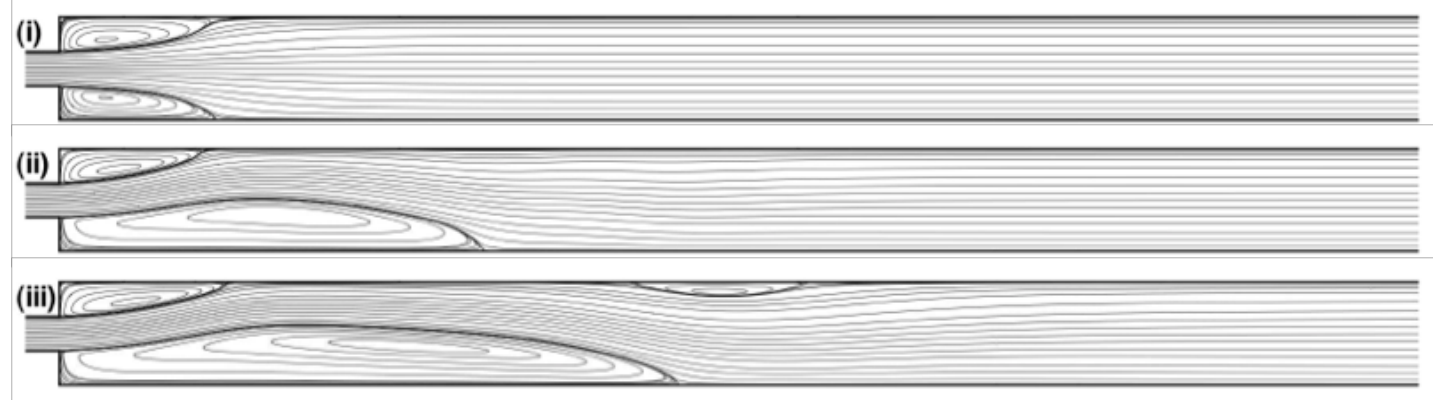

(c)
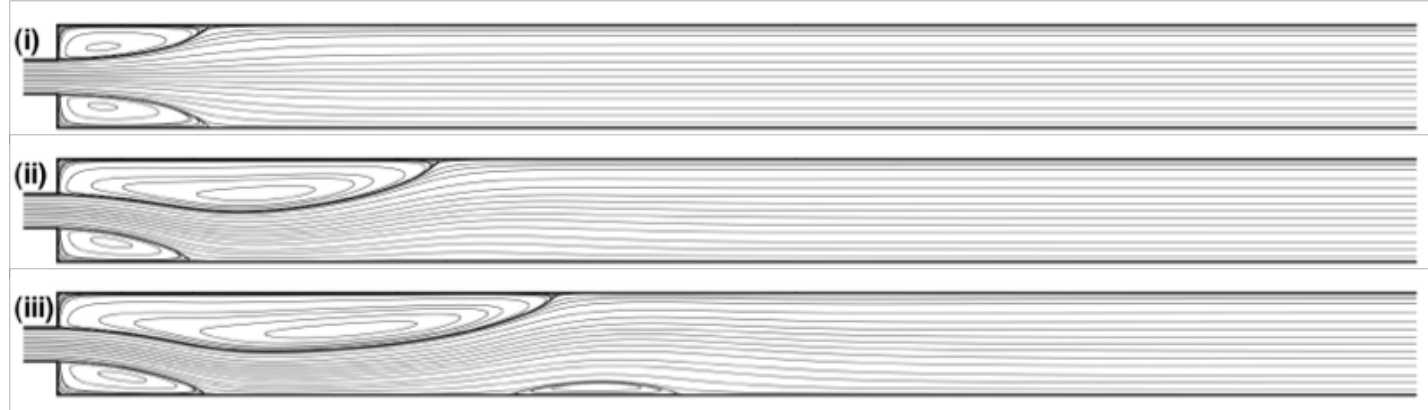

Figure 10: Flow patterns in the 1:3 planar sudden expansion at three different regimes are shown for each case: (i) symmetric flow $\left(R e_{g e n}=0.8 R e_{g e n, c r 1}\right)$; (ii) asymmetric flow $\left[R e_{g e n}=0.5\left(R e_{g e n, c r 1}+R e_{g e n, c r 2}\right)\right]$ and (iii) asymmetric flow with a third recirculating eddy $\left(R e_{g e n}=1.2 R e_{g e n, c r 2}\right)$ at different power-law index values: (a) $n=0.4$; (b) $n=0.6$; (c) $n=0.8$. 
(a)
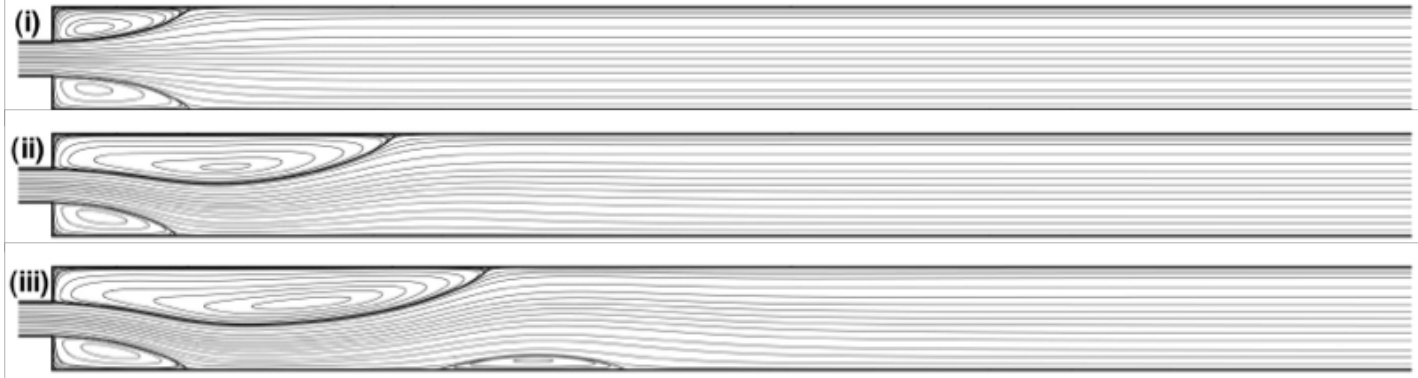

(b)
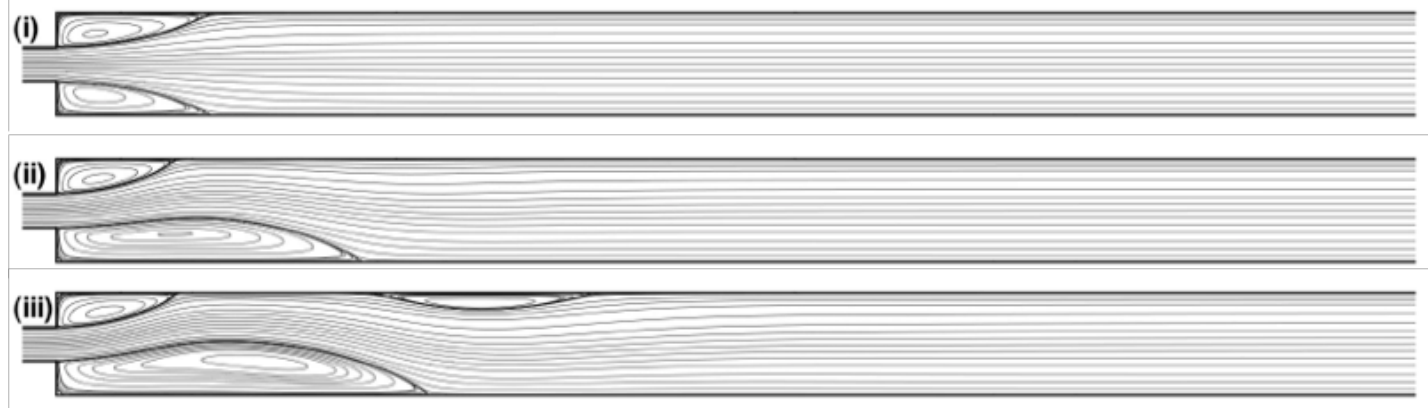

(c)
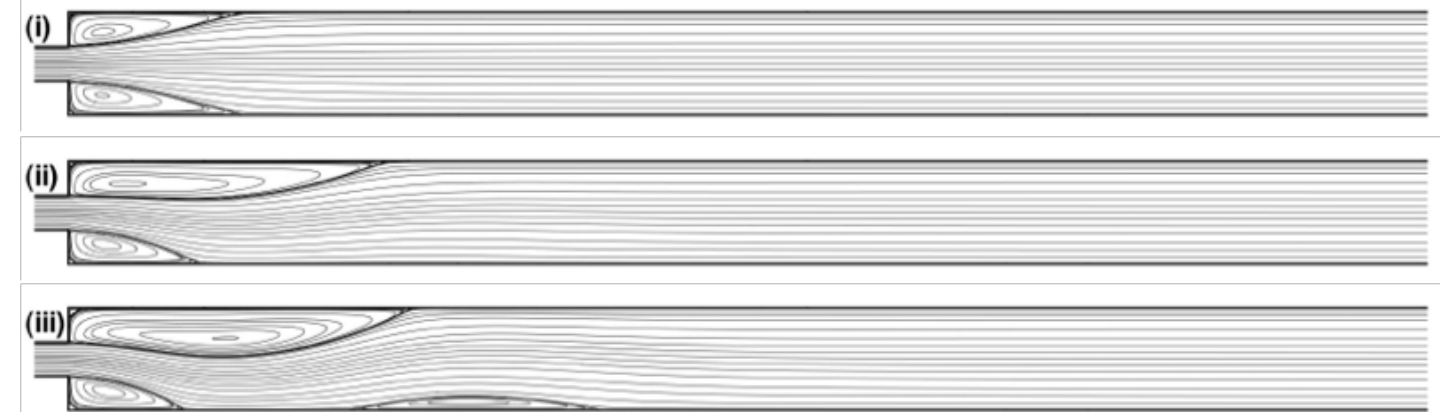

Figure 11: Flow patterns in the 1:3 planar sudden expansion at three different regimes are shown for each case: (i) symmetric flow $\left(R e_{g e n}=0.8 R e_{g e n, c r 1}\right)$; (ii) asymmetric flow $\left[R e_{g e n}=0.5\left(R e_{g e n, c r 1}+R e_{g e n, c r 2}\right)\right]$ and (iii) asymmetric flow with a third recirculating eddy $\left(\operatorname{Re}_{\text {gen }}=1.2 R e_{\text {gen }, c r 2}\right)$ at different power-law index values: (a) $n=1$; (b) $n=1.4$; (c) $n=2$. 

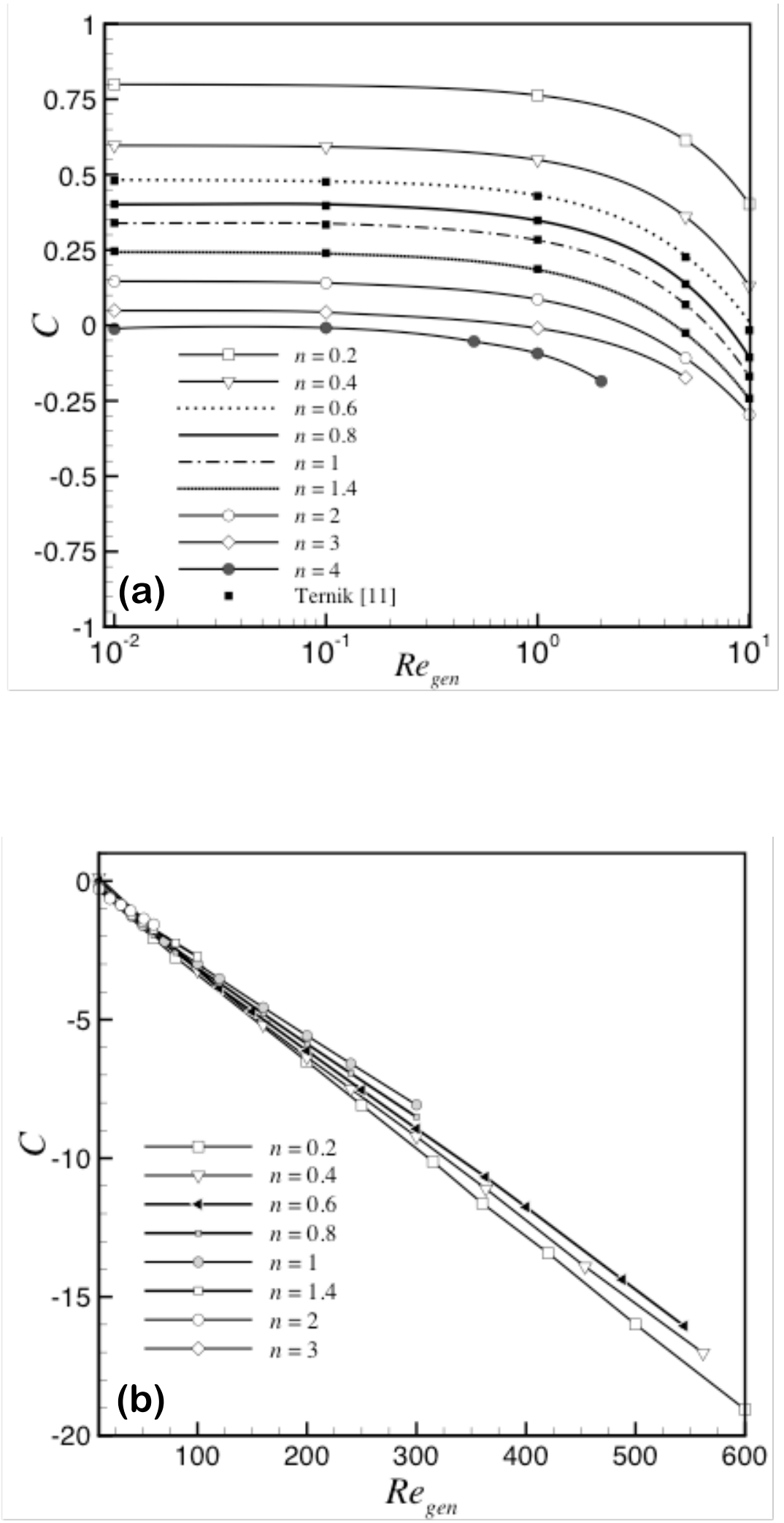

Figure 12: Variation of Couette correction, $C$, with the generalized Reynolds number $\left(R e_{g e n}\right)$ for various values of power-law index. When available, comparison with data from Ternik [12] is also presented: (a) Low range of generalized Reynolds numbers and (b) Higher range of generalized Reynolds numbers. 